\title{
High performance silicon optical modulators
}

\author{
G. T. Reed ${ }^{1}$, D. J. Thomson ${ }^{1}$, F. Y. Gardes ${ }^{1}$, Y. Hu ${ }^{1}$, N. Owens ${ }^{1}$, K. Debnath ${ }^{2}$, L. O'Faolain ${ }^{2}$, T. F. \\ Krauss $^{2}$, L. Lever ${ }^{3}$, Z. Ikonic ${ }^{3}$, R. W. Kelsall ${ }^{3}$, M. Myronov ${ }^{4}$, D. R. Leadley ${ }^{4}$ I. P. Marko ${ }^{5}$, S. J. \\ Sweeney $^{5}$, D. C. Cox $^{5}$, A. Brimont ${ }^{6}$, P. Sanchis ${ }^{6}$, G.-H. Duan ${ }^{7}$, A. Le Liepvre ${ }^{7}$, C. Jany ${ }^{7}$, M. \\ Lamponi $^{7}$, D. Make ${ }^{7}$, F. Lelarge ${ }^{7}$, J.M. Fedeli ${ }^{8}$, S. Messaoudene ${ }^{8}$, S. Keyvaninia ${ }^{9}$, G. Roelkens ${ }^{9}$, D. \\ Van Thourhout ${ }^{9}$, S. Liu ${ }^{1}$, X. Yang ${ }^{1}$ and P. Petropoulos ${ }^{1}$ \\ 1- Optoelectronics Research Centre, University of Southampton, UK. \\ 2- SUPA, School of Physics and Astronomy, University of St Andrews, UK. \\ 3- Institute of Microwaves and Photonics, University of Leeds, UK. \\ 4- Department of Physics, University of Warwick, UK. \\ 5- Advanced Technology Institute, University of Surrey, UK. \\ 6- Valencia Nanophotonics Technology Centre, Universitat Politècnica de València, Spain \\ 7- III-V Lab, a joint lab of 'Alcatel-Lucent Bell Labs France', 'Thales Research and Technology' and \\ 'CEA Leti', Campus Polytechnique, France. \\ 8-CEA, LETI, Minatec Campus, France. \\ 9- Photonics Research Group, INTEC, Ghent University-IMEC, Belgium.
}

\begin{abstract}
In this work we present results from high performance silicon optical modulators produced within the two largest silicon photonics projects in Europe; UK Silicon Photonics (UKSP) and HELIOS. Two conventional MZI based optical modulators featuring novel self-aligned fabrication processes are presented. The first is based in 400nm overlayer SOI and demonstrates $40 \mathrm{Gbit} / \mathrm{s}$ modulation with the same extinction ratio for both TE and TM polarisations, which relaxes coupling requirements to the device. The second design is based in $220 \mathrm{~nm} \mathrm{SOI}$ and demonstrates $40 \mathrm{Gbits} / \mathrm{s}$ modulation with a $10 \mathrm{~dB}$ extinction ratio as well modulation at $50 \mathrm{Gbit} / \mathrm{s}$ for the first time. A ring resonator based optical modulator, featuring FIB error correction is presented. 40Gbit/s, 32fJ/bit operation is also shown from this device which has a 6 um radius. Further to this slow light enhancement of the modulation effect is demonstrated through the use of both convention photonic crystal structures and corrugated waveguides. Fabricated conventional photonic crystal modulators have shown an enhancement factor of 8 over the fast light case. The corrugated waveguide device shows modulation efficiency down to $0.45 \mathrm{~V} . \mathrm{cm}$ compared to $2.2 \mathrm{~V} . \mathrm{cm}$ in the fast light case. $40 \mathrm{Gbit} / \mathrm{s}$ modulation is demonstrated with a $3 \mathrm{~dB}$ modulation depth from this device. Novel photonic crystal based cavity modulators are also demonstrated which offer the potential for low fibre to fibre loss. In this case preliminary modulation results at $1 \mathrm{Gbit} / \mathrm{s}$ are demonstrated. $\mathrm{Ge} / \mathrm{SiGe}$ Stark effect devices operating at $1300 \mathrm{~nm}$ are presented. Finally an integrated transmitter featuring a III-V source and MZI modulator operating at $10 \mathrm{Gbit} / \mathrm{s}$ is presented.
\end{abstract}

\section{INTRODUCTION}

The optical modulator is central to any optical communication system. The attractiveness of silicon as a material in which to build the optical circuits required to implement such systems is very much reliant on the performances of the individual components which can be formed to be sufficient for both today and tomorrows requirements. Within both the EPSRC funded UK silicon photonics (UKSP) and FP7 funded HELIOS projects there is a strong modulator activity, covering different types of optical modulator compatible with CMOS. Over the previous decade the performances of silicon optical modulators has been dramatically improving with the most impressive devices being from those based upon the plasma dispersion effect. There are three main types of structure which have been used to manipulate free carrier densities in interaction with the propagating light and thus modulate via the plasma dispersion effect. Firstly there are carrier injection based devices which use a PIN diode structure with the waveguide located in the intrinsic region. When this device is forward biased carriers are injected into the intrinsic region, changing the refractive index and

Nanophotonics and Micro/Nano Optics, edited by Zhiping Zhou, Kazumi Wada, Proc. of SPIE Vol. 8564, 85640H (c) 2012 SPIE · CCC code: 0277-786/12/\$18 - doi: 10.1117/12.2001296 
therefore the phase of the propagating light. Although the modulation produced is relatively very phase efficient and the device simple to produce, the speed is mostly limited to around a $\mathrm{GHz}$ due to the relatively long free carrier recombination lifetime in silicon. However this may be sufficient in some lower specification applications where compactness is key. Secondly there are carrier accumulation based devices such as the one which was the first to experimentally cross the $1 \mathrm{GHz}$ barrier in silicon [1]. Such devices use a thin insulating layer in the waveguide around which carriers accumulate when voltage is applied to the device. Such devices are the most complicated of the three types to produce since the thin insulating layer has to be incorporated into the guiding region. Carrier accumulation based devices do however show a good combination of high speed and high efficiency. Finally there are carrier depletion based devices which use the depletion of carriers from a PN junction located in or around the waveguide to modulate the phase of the light. Most of the devices reported in the previous few years have been based upon carrier depletion. This is most likely due to the high speed performance and the relative ease of fabrication compared with accumulation based device. A relatively low modulation efficiency however commonly results in device lengths in the millimetre regime. Within the UKSP and HELIOS projects our research group now based in the Optoelectronics Research Centre (ORC) at the University of Southampton have worked on two unique designs of phase modulator based upon carrier depletion and incorporated into Mach-Zehnder Interferometer (MZI) structures. As mentioned above carrier depletion based are relatively inefficient. The power consumption of the devices can also be problematic in a world where everyone is concerned upon energy usage and ultimately the power per bit. Within the project different methods are being developed to address this issue. Firstly there is the use of resonant structures rather than a MZI which results in vastly reduced device lengths. Next there is the use of photonic crystal structures to slow the light in the phase modulator to increase the interaction of the light with the modulating mechanism. Finally as an excursion from plasma dispersion based devices, a modulator based upon the quantum confined stark effect (QCSE) in a $\mathrm{Ge} / \mathrm{SiGe}$ quantum well structure is investigated. High performance modulation is observed in all cases. Following on from the successful development of the modulator the next step is to integrate it with other photonic components to begin to build up functional integrated photonic circuits. One particular problem in the silicon photonics is the development of the light source due the indirect bandgap in silicon. Within the HELIOS project hybrid III-V on silicon sources which use the III-V regions for gain and have the cavity within silicon are being developed. In this work we show a first integration of such a source with a MZI carrier depletion modulator.

\section{CARRIER DEPLETION MODULATORS}

\section{0nm phase modulator}

The cross-section of one variant of phase modulator which has been under development with the UKSP project is shown in figure $1[2]$.

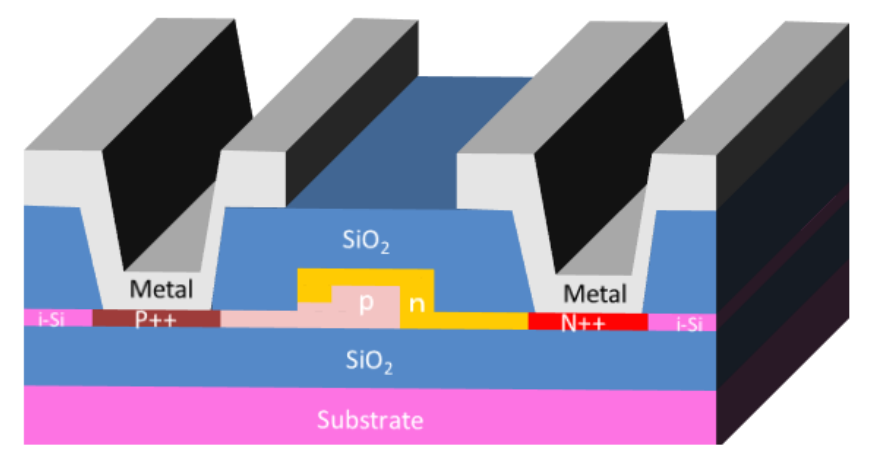

Figure 1-Cross sectional diagram of carrier depletion phase modulator with wraparound pn junction

It is based in SOI with a $400 \mathrm{~nm}$ overlayer thickness and features a wraparound PN junction structure. The waveguide therefore has a height of $400 \mathrm{~nm}$, the width is $410 \mathrm{~nm}$ and the slab height $100 \mathrm{~nm}$. The waveguide is doped mostly p-type. An n-type strip is then formed up the two sides of the rib, along the top of the rib and along the slab to one side abutting a highly doped n-type region. The slab on the other side is doped p-type and connects the rib to a highly doped p-type region. The highly doped regions allow ohmic connections to be made to the electrodes which are used to drive the device. Coplanar waveguide (CPW) travelling wave electrodes are used to co-propagate the light and driving signal. A 
key motivation for silicon photonics is its cost effectiveness. To achieve this, mass production is required with high yield. Within the design of the carrier depletion modulators fabrication simplicity has been a strong consideration. The performance of carrier depletion devices tend to be very sensitive to the alignment of the PN junction relative to the waveguide as was reported in [3]. For this reason a self-aligned process has been developed to produce the devices. The self-aligned process ensures that the positioning of the PN junction is repeatable therefore reducing performance variations and increasing yield. The device has been measured at 10Gbit/s and 40Gbit/s by applying electrical PRBS data signals to one arm of the MZI.
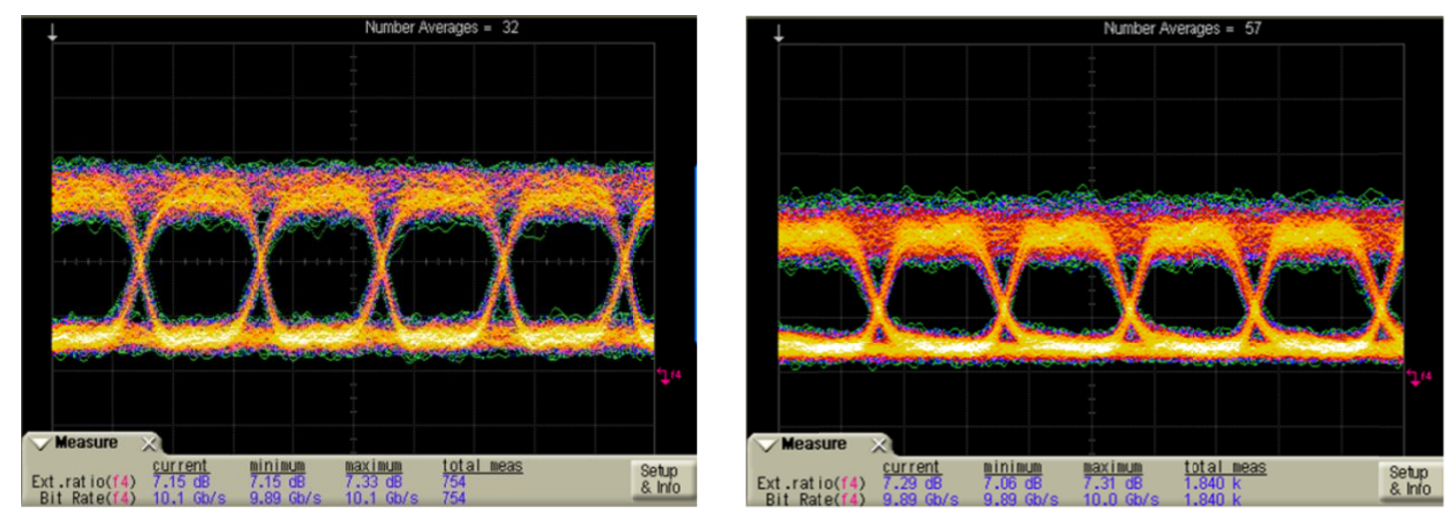

Figure 2-Optical output of the device at 10Gbit/s for TE polarisation (left) TM polarisation (right)
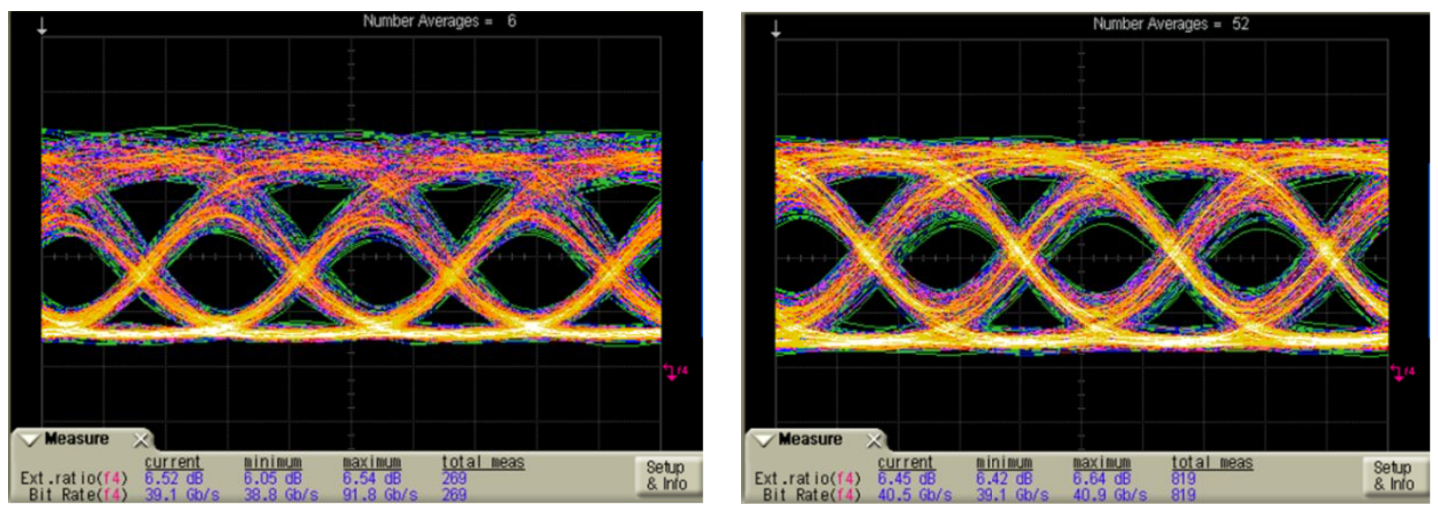

Figure 3 - Optical output of the device at 40Gbit/s for TE polarisation (left) TM polarisation (right)

The extinction ratio is approximately $7.3 \mathrm{~dB}$ for both polarisations at $10 \mathrm{Gbit} / \mathrm{s}$ and $6.5 \mathrm{~dB}$ for both polarisations at $40 \mathrm{Gbit} / \mathrm{s}$. The similarity of the performance of the device at both polarisations is an advantage as the polarisation of the coupled light to the device is less critical. In devices which employ travelling wave electrodes such as this any power not consumed by the phase modulator will be dissipated in the termination at the end of the electrode. Since the termination is essential the devices operation it's power consumption should be included in that of the overall device. The power consumption of this device has been calculated as $21.1 \mathrm{pJ} / \mathrm{bit}$ at $10 \mathrm{Gbit} / \mathrm{s}$ and $4.5 \mathrm{pJ} / \mathrm{bit}$ at $40 \mathrm{Gbit} / \mathrm{s}$.

\section{0nm phase modulator}

The cross-section of the second variant of phase modulator which has been under development with the HELIOS project is shown in figure 4 [4-6]. This device is formed in $220 \mathrm{~nm}$ overlayer SOI. The rib height is therefore $220 \mathrm{~nm}$ the slab height $100 \mathrm{~nm}$ and the waveguide width $400 \mathrm{~nm}$. The rib region and slab to one side is doped p-type. The slab region to the other side is doped n-type. The concentration of doping in the n-type region is made higher than in the p-type region such that the depletion extends mostly into the waveguide during reverse bias. The $\mathrm{n}$ and $\mathrm{p}$-type doped slab regions extend out to highly doped regions which form ohmic contacts to the coplanar waveguide electrodes. A self-aligned 
process can be used to form the PN junction. The phase modulators are incorporated into asymmetric MZI to convert the phase modulation into intensity modulation.

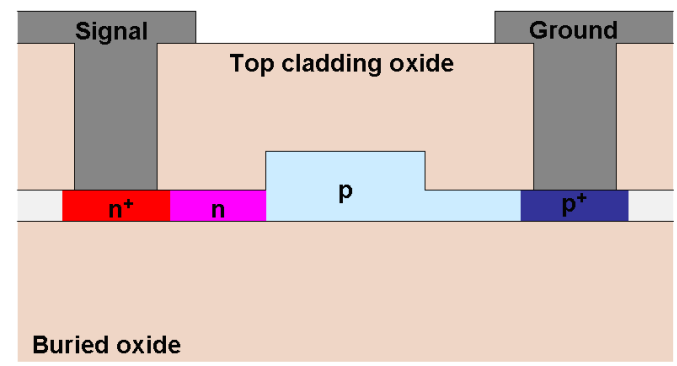

Figure 4-Cross sectional diagram of carrier depletion phase modulator self-aligned pn junction

The DC performance of an example modulator is shown in figures 5 and 6 . In figure 5 it can be seen that for the MZI with the $3.5 \mathrm{~mm}$ phase shifter a $\pi$ phase shift is achieved at around $6.5 \mathrm{~V}$.
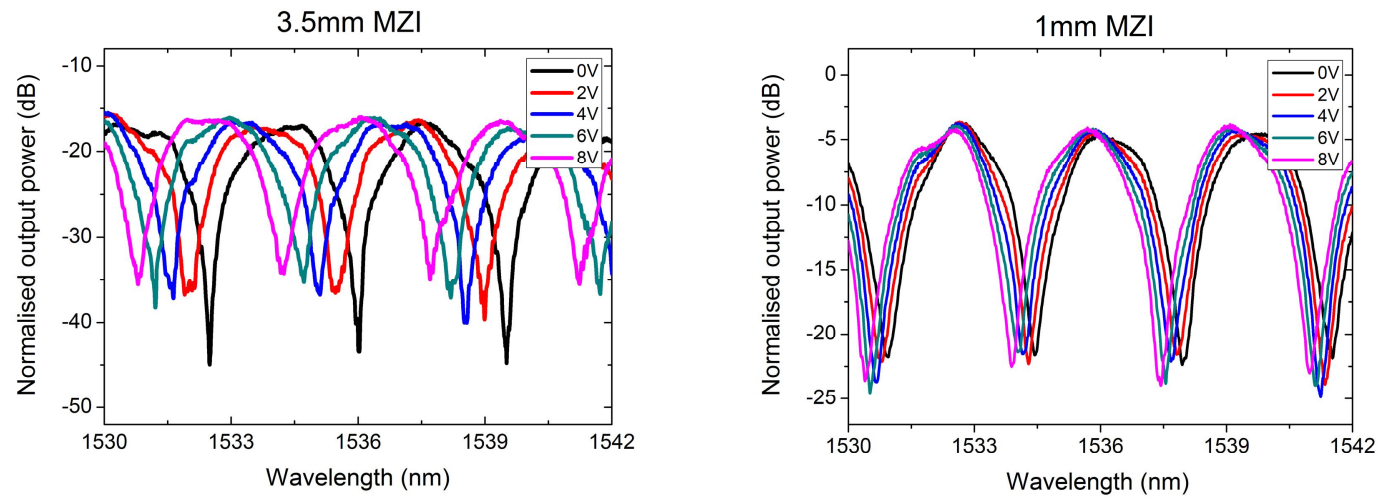

Figure 5 - Spectral response of MZI with $3.5 \mathrm{~mm}$ phase shifter (left) and $1 \mathrm{~mm}$ phase shifter (right) at different reverse bias voltages

A variation in doping across the wafer provided samples with different phase efficiencies. The best measured was $2.3 \mathrm{~V} . \mathrm{cm}$.
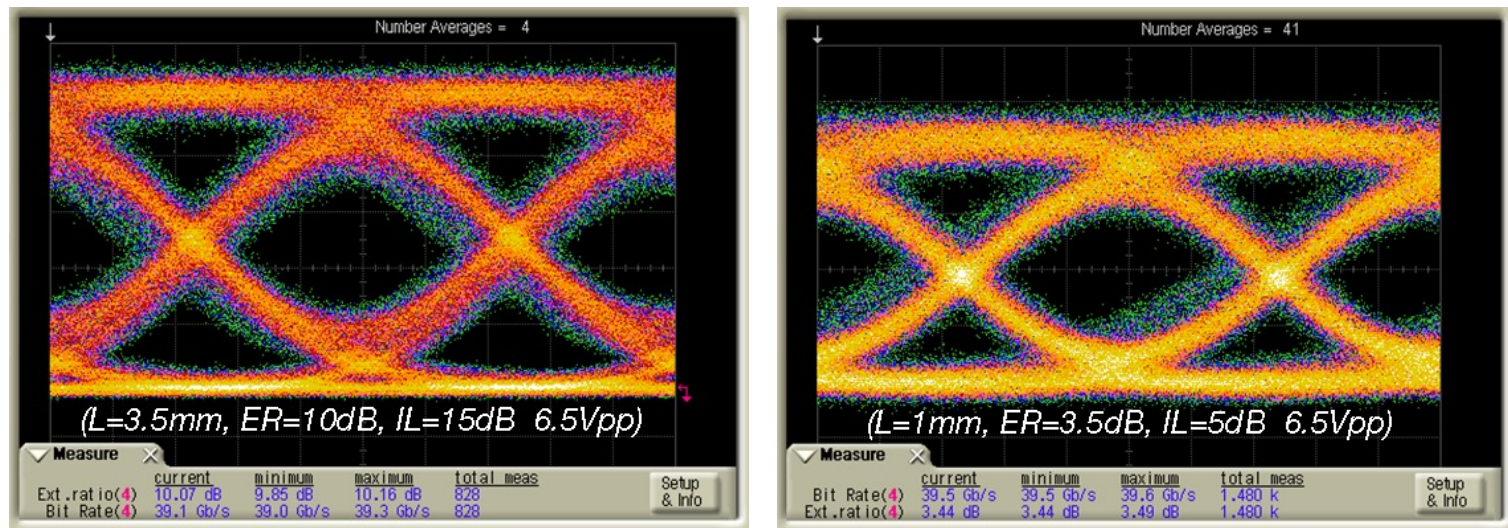

Figure 6-40Gbit/s eye diagrams from the MZI with $3.5 \mathrm{~mm}$ phase modulator (left) and $1 \mathrm{~mm}$ phase modulator (right)

The high speed performance of the device was also analysed in the same way as the previous carrier depletion device but at $40 \mathrm{Gbit} / \mathrm{s}$ (Figure 6) and 50Gbit/s (Figure 7). At 40Gbit/s (6.5V peak to peak) an open eye diagram with a modulation depth of $10 \mathrm{~dB}$ is observed from the MZI with $3.5 \mathrm{~mm}$ MZI. With the shorter device the modulation depth is reduced to $3.5 \mathrm{~dB}$. At $50 \mathrm{Gbit} / \mathrm{s}$ the modulation depth from the MZI with $1 \mathrm{~mm}$ phase shifter is reduced slightly to $3 \mathrm{~dB}$. In both cases the peak to peak drive voltage was $6.5 \mathrm{~V}$. 


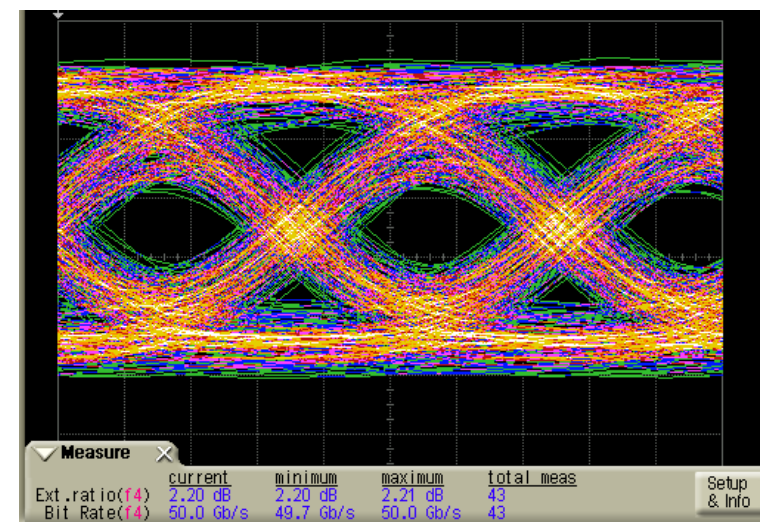

Figure 7 -50Gbit/s eye diagrams from the MZI with $1 \mathrm{~mm}$ phase modulator

Using the same method to calculate the power consumption as with the previous device gives $5.3 \mathrm{pJ} / \mathrm{bit}$ at $40 \mathrm{Gbit} / \mathrm{s}$ and $4.2 \mathrm{pJ} / \mathrm{bit}$ at $50 \mathrm{Gbit} / \mathrm{s}$. The chirp of the device has also been theoretically and experimentally characterised for both singledrive and dual-drive schemes. It has been shown that for the dual-drive case the chirp is very small showing the potential for the implementation of silicon based optical modulators into longer haul applications. Within the HELIOS project there is a further phase modulator design investigated by partners from University Paris-Sud [7]. This design has also demonstrated excellent performance.

\section{MODULATION ENHANCEMENT}

In order reduce the footprint and/or the drive voltage (and therefore power consumption) of the phase modulators different methods of enhancing the modulation effect to that of a conventional MZI approach have been investigated. These include using a ring resonator structure, a corrugated waveguide slow light approach, a dispersion engineered photonic crystal slow light approach and using a photonic crystal based cavity structure.

\section{Ring resonator modulator}

The carrier depletion phase modulator based in $220 \mathrm{~nm}$ SOI (figure 4) has also been incorporated into a ring resonator structure as shown in figure 8 [8]. The $\mathrm{p}+$ contact is in the centre of the ring resonator and the $\mathrm{n}+$ region surrounds it. The ring radius is 6um and the separation between the ring and the access waveguide $150 \mathrm{~nm}$ at the closest point. Since the PN junction is at the outer edge of the ring waveguide the light has a greater interaction with the depletion region than it does in a straight waveguide as used in the in the convention MZI. Potentially this phase modulator design is therefore better suited for implementation in ring resonator structures than MZI. The ring modulator has been tested at high speed in a similar manner as the conventional MZI except that it is driven as a lumped element. Since the impedance of the ring modulator is not $50 \mathrm{ohm}$ a broadband RF attenuator is inserted between the device and the driver to avoid reflections which can both degrade the signal and damage the driver. This reduces the peak to peak drive voltage to $1.4 \mathrm{~V}$. Optical eye diagrams at $30 \mathrm{Gbit} / \mathrm{s}$ and $40 \mathrm{Gbit} / \mathrm{s}$ are shown in figure 9 . The modulation depth at either speed is $1.6 \mathrm{~dB}$ and $1.1 \mathrm{~dB}$ respectively. The reduction in modulation depth between $30 \mathrm{Gbit} / \mathrm{s}$ and $40 \mathrm{Gbit} / \mathrm{s}$ can be mostly explained by the imperfect drive signal to the device. The reason for the low modulation depth is the combination of a low Q (1015), low drive voltage and small ring radius. The modulation depth can is best improved by improving the Q. Our analysis suggests that significant improvements can be made whilst maintaining 40Gbit/s operation. The power consumption of the device taking into account that the voltage at the ring could be as much as double due to the reflection of the drive signal is $32 \mathrm{fJ} / \mathrm{bit}$. It can be seen that this is much lower than with the MZI based modulators, however, no account is taken any power required to stabilise the temperature of the device. 


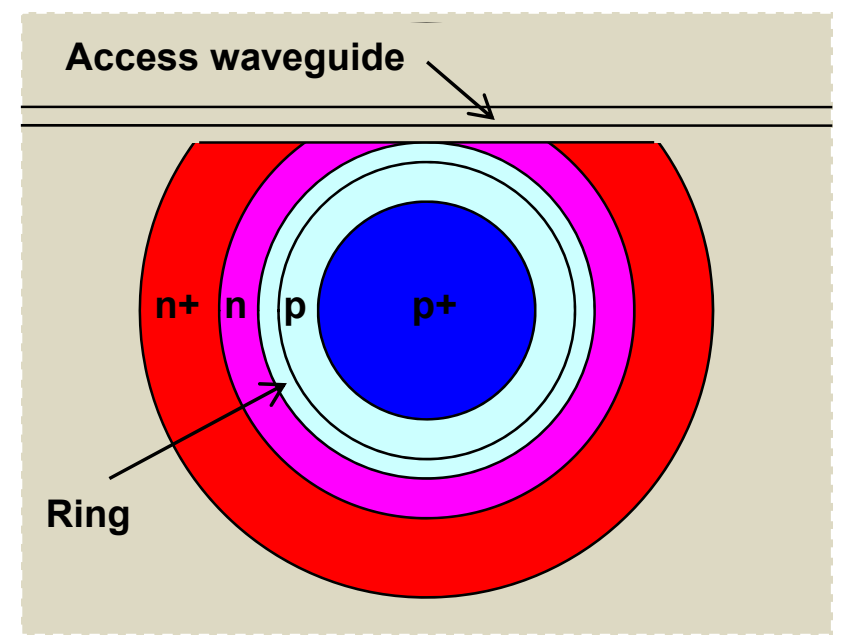

Figure 8 - Plan diagram of the ring resonator modulator
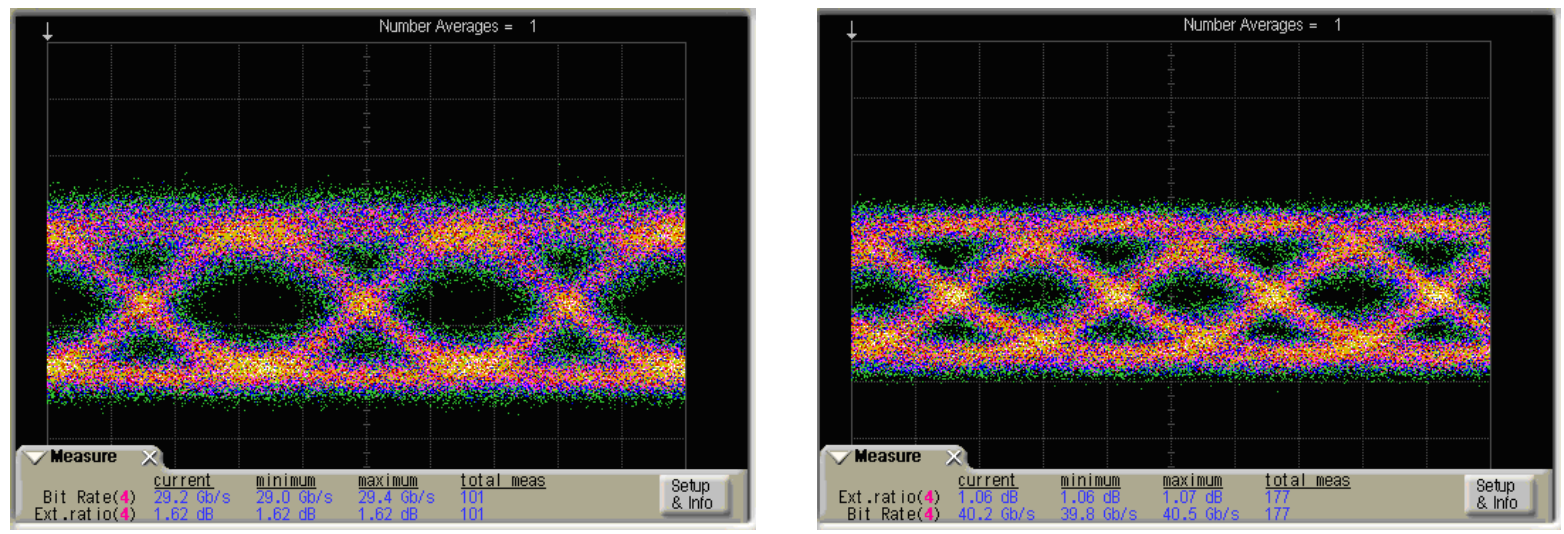

Figure $9-50$ Gbit/s eye diagrams from the MZI with $1 \mathrm{~mm}$ phase modulator

\section{Corrugated waveguide modulator}

By slowing the light propagating through the modulator a greater interaction of the light with the modulating effect is achieved. Within the HELIOS project slow light propagation is achieved through the use of a one-dimensional (1D) periodic structure consisting of a laterally corrugated waveguide [9-10]. The corrugated waveguide is based in $220 \mathrm{~nm}$ overlayer SOI and features a central PN junction as shown in figure 10.

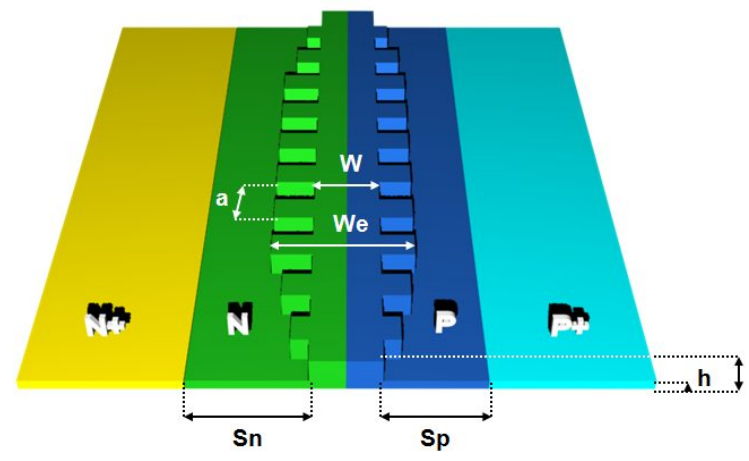

Figure 10-Diagram of corrugated waveguide modulator

The width of the waveguide (W) is $300 \mathrm{~nm}$ and width of the corrugations $\left(\mathrm{W}_{\mathrm{e}}\right)$ is $650 \mathrm{~nm}$. The waveguide and slab heights are $220 \mathrm{~nm}$ and $100 \mathrm{~nm}$ respectively. The period of the corrugation (a) is $310 \mathrm{~nm}$. Phase modulators of length just $1000 \mathrm{um}$ 
and 500um are inserted into asymmetric MZIs. The MZI with 1000um phase modulator is operated with a group index of $\sim 9.5$ to provide a phase efficiency of $0.6 \mathrm{~V} . \mathrm{cm}$. A $10 \mathrm{Gbit} / \mathrm{s}$ drive signal with a drive voltage of just $2 \mathrm{~V}$ peak to peak is applied to the device. An open eye diagram with modulation depth of $4.3 \mathrm{~dB}$ results (figure 11 left). The MZI with 500um phase modulator, operated with a group index of $\sim 8$, has a $5 \mathrm{~V}$ peak to peak, $40 \mathrm{Gbit} / \mathrm{s}$ drive signal applied. An open eye diagram with modulation depth of $6.6 \mathrm{~dB}$ results (figure 11 right).
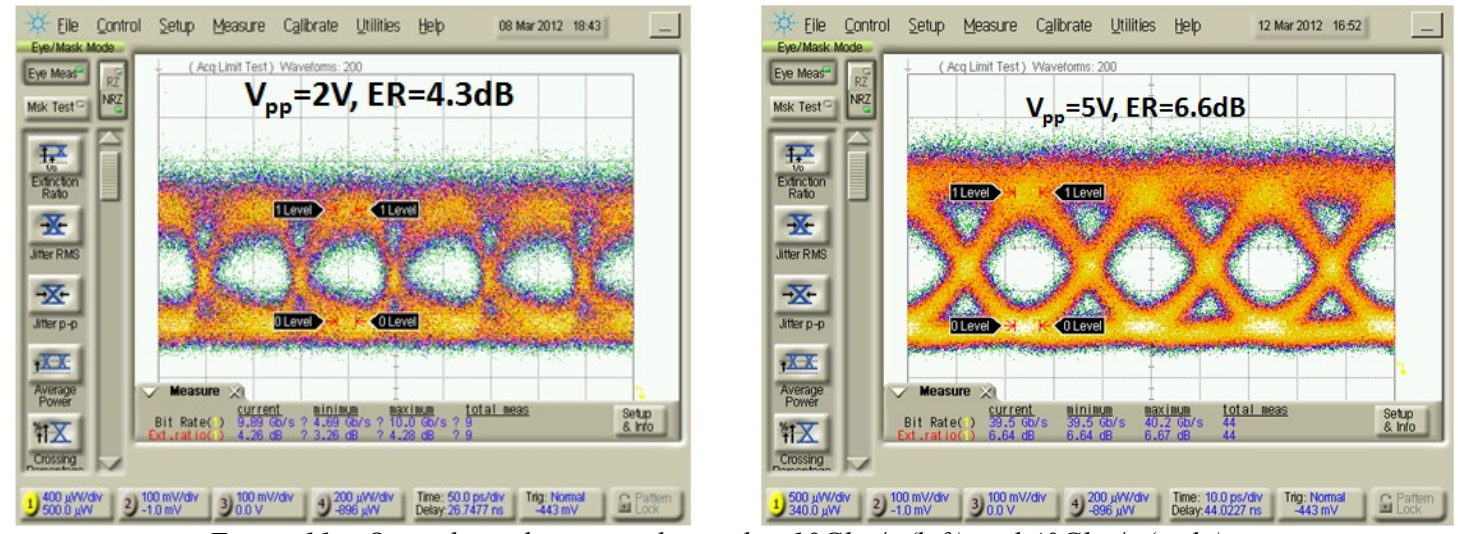

Figure 11 - Optical eye diagrams obtained at 10Gbit/s (left) and 40Gbit/s (right)

In terms of the power consumption, these devices still require a termination so the same calculation holds as with the conventional MZI however, the reduction in the required drive voltage allows for a reduction in power consumption. In the $10 \mathrm{Gbit} / \mathrm{s}$ case the power consumption is $2 \mathrm{pJ} / \mathrm{bit}$ and $3.1 \mathrm{pJ} / \mathrm{bit}$ at $40 \mathrm{Gbit} / \mathrm{s}$.

\section{Dispersion engineered photonic crystal modulator}

To further demonstrate the potential of using slow light to enhance the modulation efficieincy a dispersion engineered photonic crystal modulator based upon carrier injection has been developed within the UKSP project [11]. SEM images of the device can be seen in figure 12 .
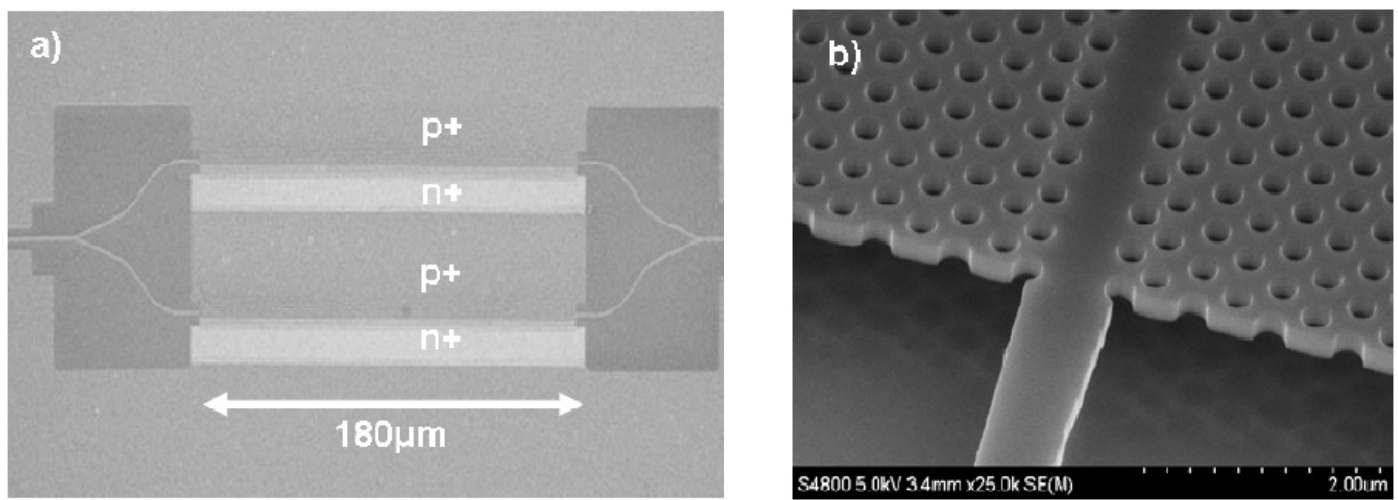

Figure 12 - Annotated plan SEM images of the dispersion engineered photonic crystal modulator

180um phase modulators have been inserted into both arms of a MZI. The normalised optical output against drive power (to one MZI arm) for two different group index values is shown in figure 13. With a group index of $\sim 720 \mathrm{~mW}$ is required to switch the device. When the device is operated further into the slow light region with a group index $\sim 30$, switching occurs with a power of approximately $4 \mathrm{~mW}$ indicating an enhancement factor around 5. Note however that the group index of 7 is larger than that of a regular silicon rib waveguide so the true enhancement factor is around 8 . This clearly demonstrates the potential to reduce the power required to drive the device through the use of slow light. 


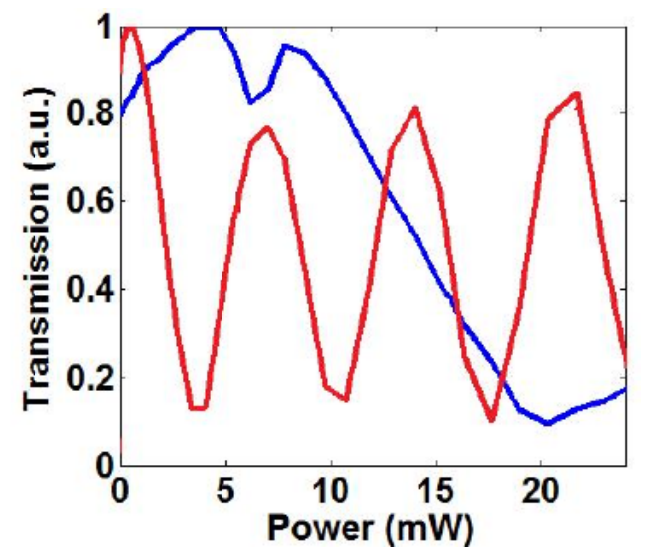

Figure 13 - Optical transmission through the device against power for group indices of $\sim 7$ (blue) and $\sim 30$ (red)

\section{Photonic crystal cavity modulator}

An alternative approach to enhance modulation using photonic crystals is to create a resonant cavity which is modulated. A multi-cavity design explored within UKSP is shown in figure 14 [12]. The cavities are vertically coupled from a large, low index contrast bus waveguide (in this case SU8) which has potential for very low coupling losses from optical fibre $(<3 \mathrm{~dB}$ experimentally realised). As a first demonstration the cavity is modulated using a carrier injection structure. Five cavities have been cascaded along the bus waveguide as shown in the right of figure 14 along with the spectral response of the device.

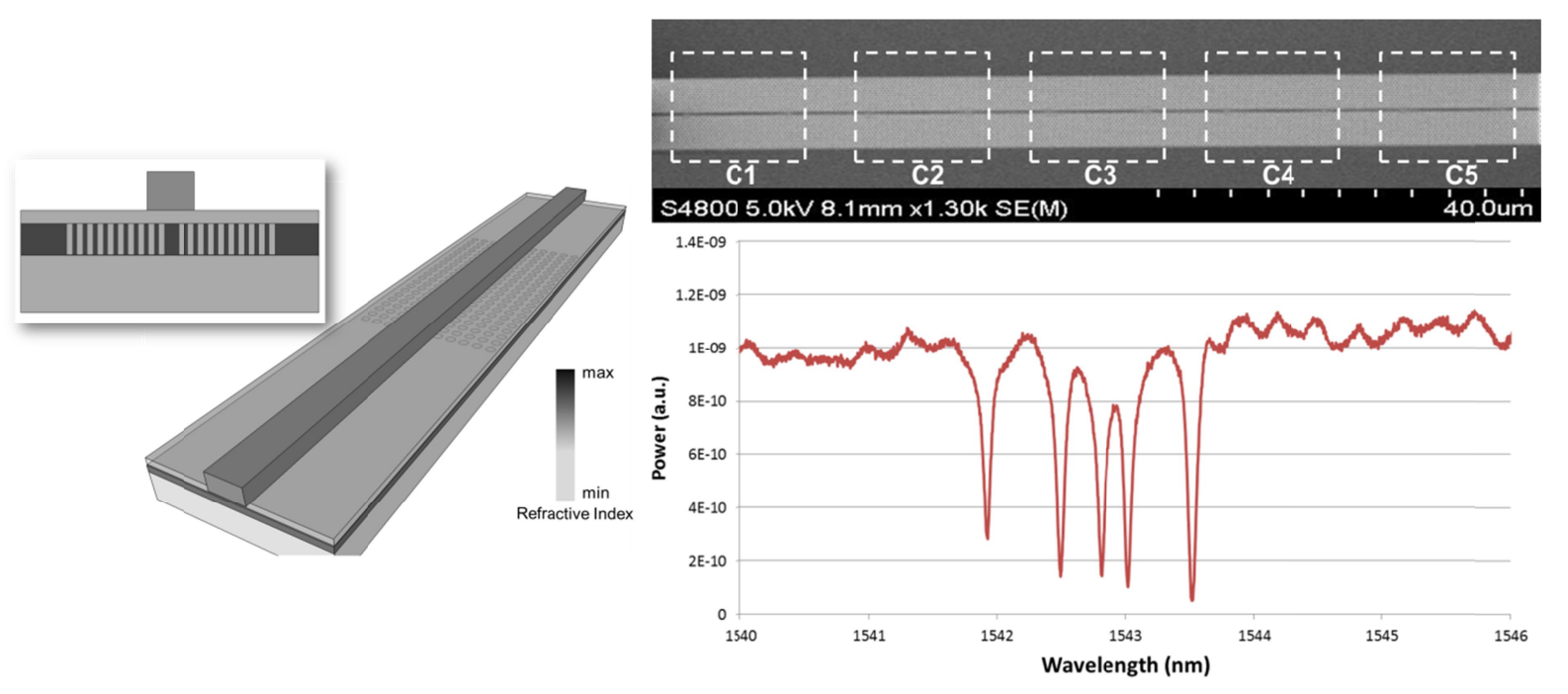

Figure 14 - Diagram of single photonic crystal cavity (left), plan SEM image of 5 cascaded cavities (top right) and optical transmission through bus waveguide (bottom right)

Figure 15 shows the DC and high speed modulation of the device. At DC the heating caused during carrier injection causes a red shift of the resonance. It can be seen that it is possible to shift the response of a single cavity whilst having little effect on adjacent cavities which are in close proximity showing potential for a large packing density. The devices have also been tested at high speed in a similar manner as the ring resonator carrier depletion modulator and have demonstrated an open eye diagram at $1 \mathrm{Gbit} / \mathrm{s}$. The speed of the device is limited in this case by the contacts. 

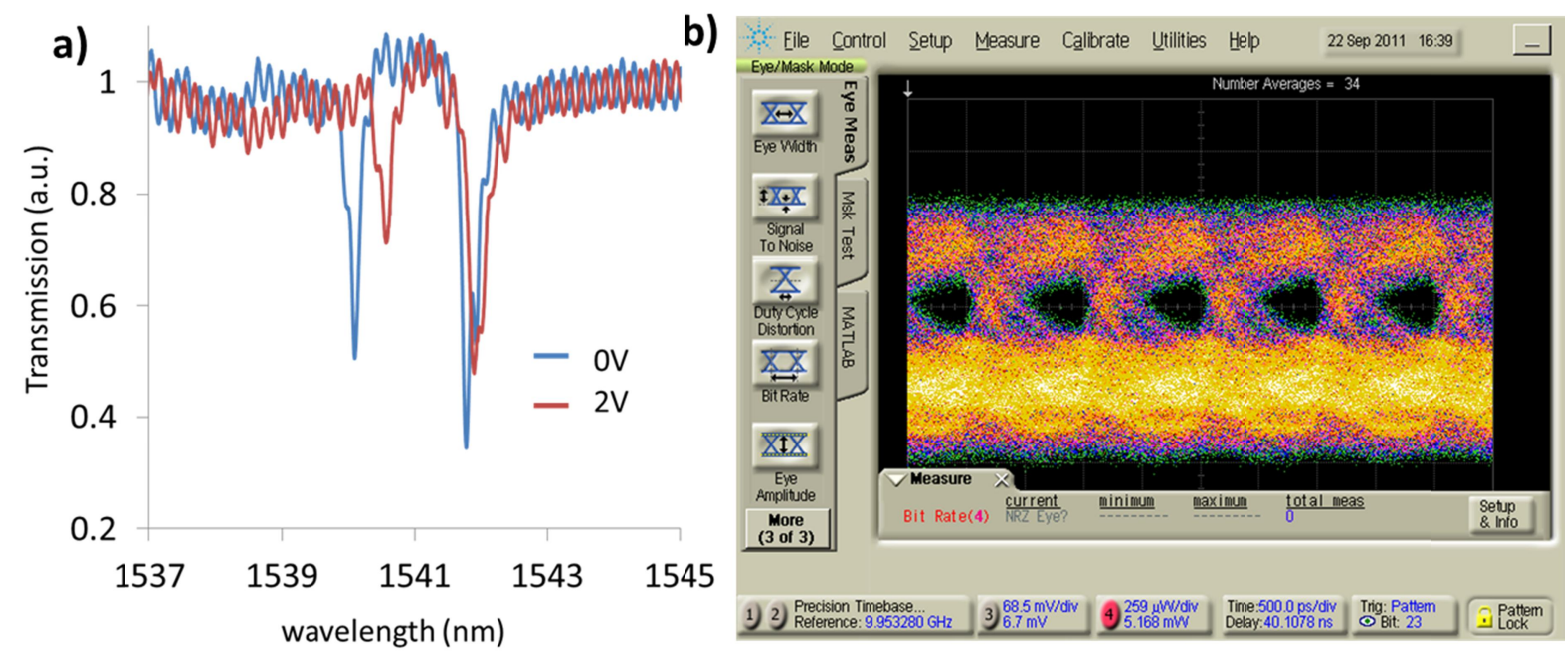

Figure 15 - Spectral response of the device with a $0 \mathrm{~V}$ and $2 \mathrm{~V}$ forward bias applied to one of the cavity modulators (left) and optical eye diagram at 1Gbit/s (right)

Multiwavelength light sources have huge potential in DWDM networks. To demonstrate the full potential of our approach, we used a multi-wavelength comb laser source with $50 \mathrm{GHz}$ channel spacing [13] shown in the inset of Fig. 5. The resonance wavelength of each of the five cavities was actively tuned by adjusting the DC bias to match five consecutive lines of the comb laser as shown in Fig. 15(b). The blue curve shows the output spectrum of the waveguide when none of the cavity wavelengths are aligned with the lines of the laser (each spike was normalized to unity for clarity) and the red curve shows the same spectrum when the cavities are aligned to the lines of the comb laser. To our knowledge, this is the first demonstration of modulation based on cascaded resonators and a comb laser.

(a)

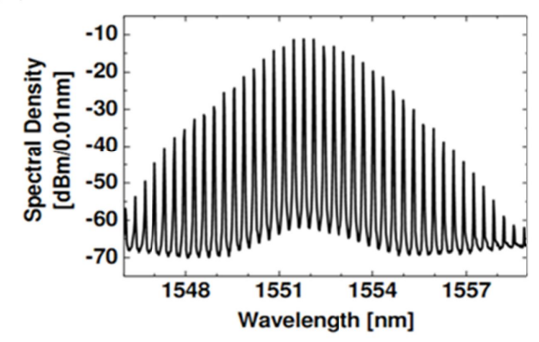

(b)

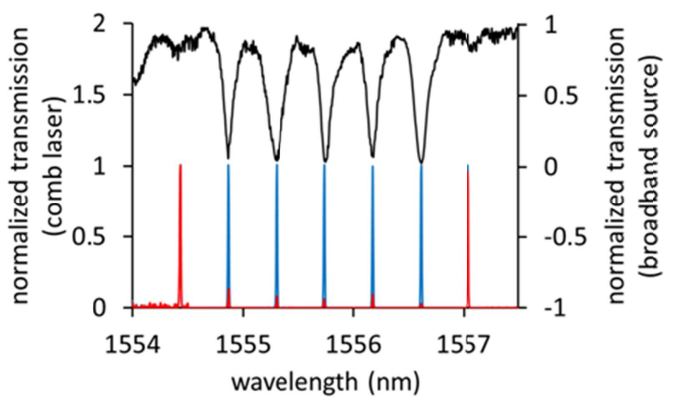

Fig. 16. (a) Optical spectrum of the multi-wavelength comb laser, (b) Black curve represents normalized transmission spectrum of the bus waveguide with a tunable laser when each cavity is actively tuned to match the comb laser spectrum. B) The normalized transmission spectra of comb laser with cavities aligned to the lines of the comb (red)- off states and misaligned (red) - the on states.

\section{QUANTUM CONFINED STARK EFFECT DEVICE}

Quantum confined stark effect (QCSE) devices are based upon the shift in the absorption edge of a quantum well heterostructure in response to an electric field applied perpendicular to the plane of the quantum wells. Within UKSP, project partners are aiming to use this effect for electroabsorption modulation [14]. Here modulation of the absorption coefficient at $1.3 \mu \mathrm{m}$ in $\mathrm{Ge} / \mathrm{SiGe}$ multiple quantum well heterostructures on silicon is reported. A cross-sectional TEM of the quantum well structure is shown in figure 17. The Ge quantum wells of $9 \mathrm{~nm}$ thick were grown on a relaxed $\mathrm{Si}_{0.22} \mathrm{Ge}_{0.78}$ buffer layer using a reverse-linear grading (RLG) technique to achieve the desired alloy composition from a pure Ge seed layer, and vertical PIN diodes were formed using reactive-ion etching. The absorption spectra were inferred from the wavelength-dependent photocurrent, which was measured using a $100 \mathrm{~W}$ xenon light source with a $3 \mathrm{~nm}$ bandwidth monochromator. 

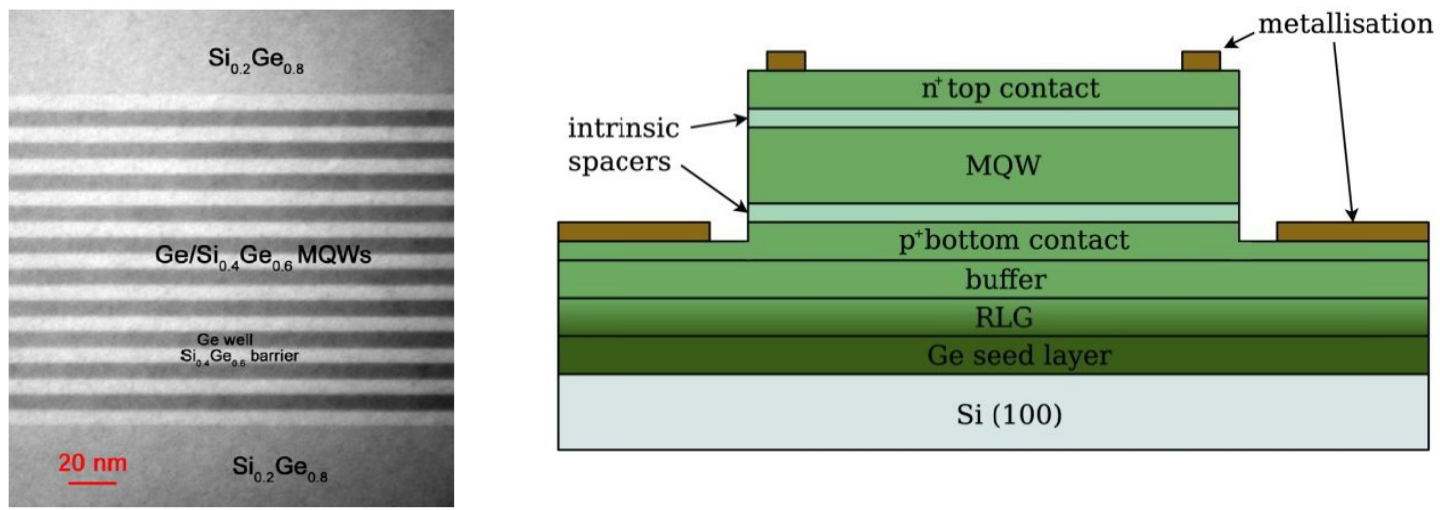

Figure 17 - Annotated cross-sectional TEM of the quantum well structure (left) and schematic diagram of the vertical p-i-n diodes (right)

Figure 18 shows the photocurrent at a range of applied biases and the ratio of the absorption coefficient compared to the $0 \mathrm{~V}$ case. It can be seen that the ratio of the absorption coefficient at $9 \mathrm{~V}$ is 3.6 at $1300 \mathrm{~nm}$ and greater than 3.2 throughout the range $1290-1315 \mathrm{~nm}$.
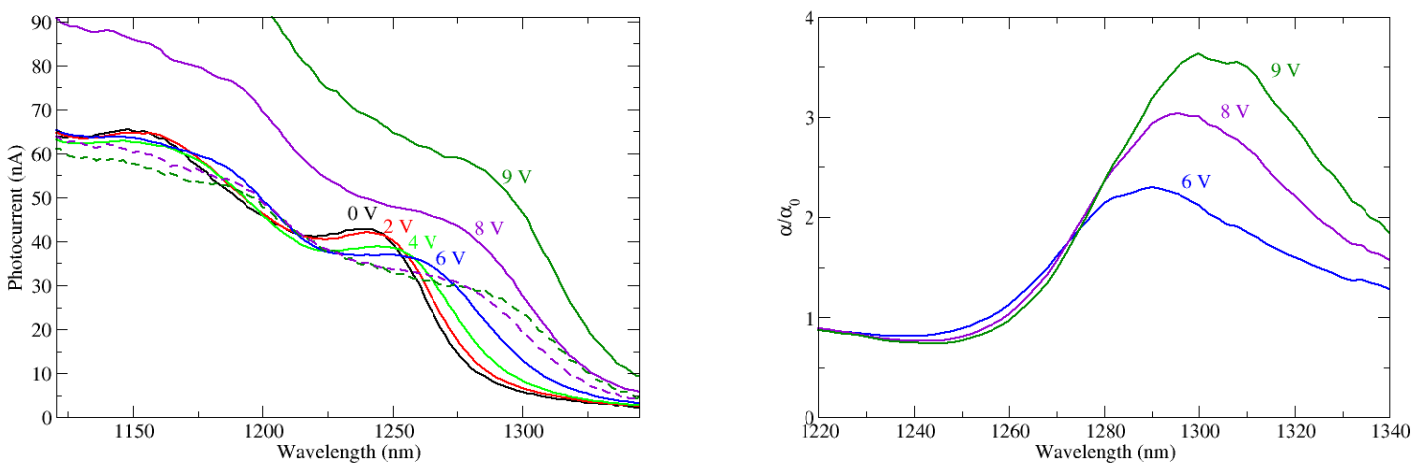

Figure 18 - Measured photocurrent against wavelength for different bias voltages (left) and ratio of the absorption coefficient compared to the $0 \mathrm{~V}$ case (right)

\section{TUNABLE TRANSMITTER}

Finally we demonstrate a tunable transmitter developed within the HELIOS project, integrating a hybrid III-V/Si laser fabricated by wafer bonding and a silicon MZI modulator [15]. Figure 19 shows a diagram of the transmitter layout and an optical microscope image of a fabricated device. The transmitter comprises a single mode hybrid III-V/silicon laser, $220 \mathrm{~nm}$ carrier depletion based MZI modulator similar to that described above and an optical output coupler. The singlemode hybrid laser includes an $\mathrm{InP}$ waveguide providing light amplification, and a SOI based ring resonator (RR) allowing single mode operation. The laser cavity is positioned in the silicon waveguiding layer and produced by etching two Bragg reflectors into the silicon waveguides.
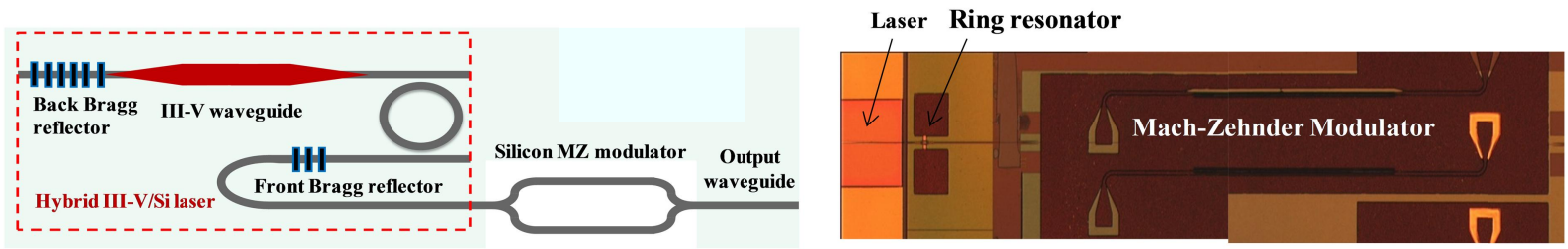

Figure 19 - Diagram of transmitter layout (left) and optical microscope image of fabricated device (right) 
The integrated lasers feature $9 \mathrm{~nm}$ tunability invoked by heating the ring resonator within the laser cavity. Phase modulators of length $3 \mathrm{~mm}$ are placed in both arms of an assymetric MZI. By tuning the laser wavelength the modulators operating point is therefore changed. Figure 20 left shows the electro optic bandwidth of the modulator at different DC biases. The modulator bandwidth increases with increasing reverse bias and is in excess of $13 \mathrm{GHz}$ for biases of $2 \mathrm{~V}$ and above. Such a modulation response guarantees operation at $10 \mathrm{Gbit} / \mathrm{s}$, and should allow modulation at data rates up to 25 $\mathrm{Gbit} / \mathrm{s}$. For the BER and eye measurements one arm of the modulator is driven with a voltage swing of around $7 \mathrm{~V}$, at 10 $\mathrm{Gb} / \mathrm{s}$ using a pseudo-random binary sequence (PRBS). Figure 20 (middle) shows the BER curves for all the wavelengths and also a reference curve for a directly modulated laser. Figure 20 (right) shows the corresponding eye diagram for all the corresponding channels. The modulation depth for each channel varies from 6 to $10 \mathrm{~dB}$, while the ER for the reference is only $4 \mathrm{~dB}$. All channels have superior BER performance than the reference for received power levels lower than $-25 \mathrm{dBm}$, due to the higher ER of the silicon modulator compared to that of the reference. For power levels higher than $-25 \mathrm{dBm}$, channels $2,3,4$ and 5 behave slightly better than the reference, achieving error free operation with BER $<$ $10^{-9}$. Other channels have minimum BER between $10^{-7}$ and $10^{-8}$, mainly limited by the optical signal to noise ratio (OSNR) due to the high coupling losses between the output waveguide and the used lensed fiber.
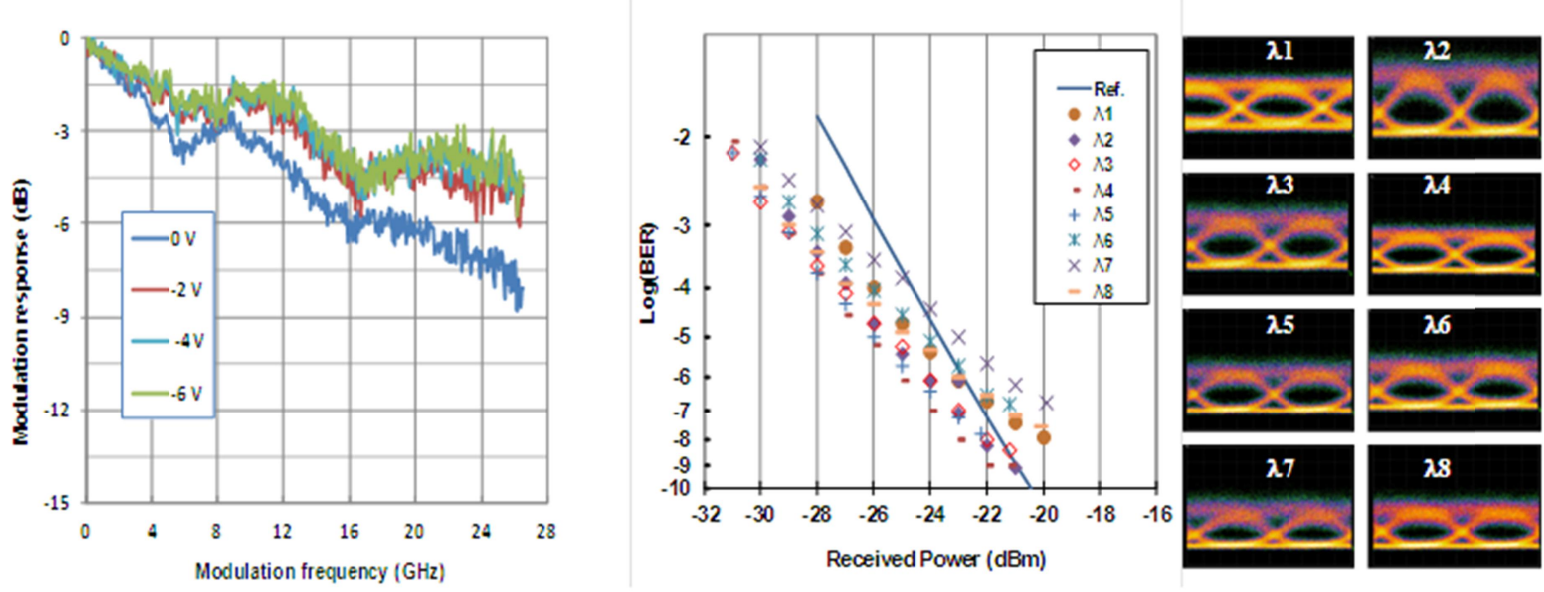

Figure 20 - Electro-optic bandwidth of the modulator at different DC bias levels (left), bit error rate measurement results (middle) and optical eye diagrams (right).

\section{CONCLUSIONS}

Several different silicon optical modulators have been presented in this work. Conventional carrier depletion, MZI based devices operating up to $50 \mathrm{Gbit} / \mathrm{s}$, or $40 \mathrm{Gbit} / \mathrm{s}$ for both polarisations has been demonstrated. $40 \mathrm{Gbit} / \mathrm{s}$ modulation has also been achieved from carrier depletion phase modulator incorporated into a ring resonator structure. Slow light enhancement via dispersion engineered photonic crystal structures and corrugated waveguides has been successfully explored. Both large enhancement factors and high speed modulation have been shown. A highly compact multichannel photonic crystal cavity based modulator has been demonstrated. Modulation of the absorption spectra of a Ge/SiGe based quantum confined stark effect device at $1300 \mathrm{~nm}$ has been presented. Finally the successful integration of a III-V laser and MZI based carrier depletion modulator operating at 10Gbit/s has been demonstrated.

\section{REFERENCES}

[1] - A. Liu, R. Jones, L. Liao, D. Samara-Rubio, D. Rubin, O. Cohen, R. Nicolaescu, and M. Paniccia, "A high-speed silicon optical modulator based on a metal-oxide-semiconductor capacitor," Nature, vol. 427, pp. 615-618, 2004.

[2] - F. Y. Gardes, D. J. Thomson, N. G. Emerson, and G. T. Reed, "40 Gb/s silicon photonics modulator for TE and TM polarisations," Opt. Express, 19(12), pp. 11804-11814, 2011 
[3] - F. Y. Gardes, A. Brimont, P. Sanchis, G. Rasigade, D. Marris-Morini, L. O'Faolain, F. Dong, J-M. Fedeli, P. Dumon, L. Vivien, T. F. Krauss, G. T. Reed and J. Martí, "High-speed modulation of a compact silicon ring resonator based on a reverse-biased pn diode," Opt. Express, 17(24), 21986-21991, (2009).

[4] - D. J. Thomson, F. Y. Gardes, G. T. Reed, F. Milesi, and J-M Fedeli, "High speed silicon optical modulator with self aligned fabrication process," Opt. Express, 18(18), 19064-19069, (2010).

[5] - D. J. Thomson, F. Y. Gardes, Y. Hu, G. Mashanovich, M. Fournier, P. Grosse, J-M. Fedeli and G. T. Reed, "High contrast 40Gbit/s optical modulation in silicon," Opt. Express, 19(12), 11507 - 11516, (2011).

[6] - D. J. Thomson, F. Y. Gardes, J-M. Fedeli, S. Zlatanovic, Y. Hu, B. P. -P. Kuo, E. Myslivets, N. Alic, S. Radic, G. Z. Mashanovich and G. T. Reed, "50Gbit/s Silicon Optical Modulator," IEEE Photonics Technology Letters, vol. 24, pp. 234-236, 2012.

[7] - M. Ziebell, D. Marris-Morini, G. Rasigade, J-M Fédéli, E. Cassan, D. Bouville and L. Vivien, "40Gbit/s low-loss silicon optical modulator based on a pipin diode", Optics Express vol. 20, Issue 10, pp. 10591-10596, 2012.

[8] - D. J. Thomson, F. Y. Gardes, D. C. Cox, J-M. Fedeli, G. Z. Mashanovich and G. T. Reed, "An alignment tolerant high speed ring resonator based silicon optical modulator," IEEE Group IV Photonics 2012.

[9] - A. Brimont, D. J. Thomson, P. Sanchis, J. Herrera, F. Y. Gardes, J. M. Fedeli, G. T. Reed, and J. Marti, "High speed silicon electro-optical modulators enhanced via slow light propagation," Optics Express, vol. 19, pp. 20876-20885, 2011.

[10] - A. Brimont, D. J. Thomson, F.Y. Gardes, J. M. Fedeli, G. T. Reed, J. Martí and P. Sanchis, "Low drive voltage 10 $\mathrm{Gb} / \mathrm{s}$ and high contrast $40 \mathrm{~Gb} / \mathrm{s}$ silicon slow wave modulators," IEEE Group IV Photonics 2012.

[11] - K. Debnath, L. O'Faolain and T. F. Krauss, "Slowlight enhanced photonic crystal modulators," IEEE Group IV Photonics 2011.

[12] - K. Debnath, L. O'Faolain, F. Gardes, D. Thomson, G. Reed and T. F. Krauss, "Low insertion loss modulator based on a vertically coupled photonic crystal resonator," SPIE Photonics West 2012.

[13] - B. Huettl, R. Kaiser, W. Rehbein, H. Stolpe, Ch. Kindel, S. Fidorra, A. Steffan, A. Umbachl and H. Heidrich, "Low noise monolithic $40 \mathrm{GHz}$ mode-locked DBR lasers based on GaInAsP/InP," International Conference on Indium Phosphide and Related Materials, 633-636, 2005.

[13] - L. Lever, Y. Hu, M. Myronov, X. Liu, N. Owens, F. Y. Gardes, I. P. Marko, S. J. Sweeney, Z. Ikonic, D. R. Leadley, G. T. Reed, and R. W. Kelsall, "Modulation of the absorption coefficient at $1.3 \mathrm{um}$ in $\mathrm{Ge} / \mathrm{SiGe}$ multiple quantum well heterostructures on silicon," Optics Letters, vol. 36, pp. 4158-4160, 2011.

[14] - G-.H. Duan, C. Jany, A. Le Liepvre, J.-G. Provost, D. Make, F. Lelarge, M. Lamponi, F. Poingt, J.-M. Fedeli, S. Messaoudene, D. Bordel, S. Brision, S. Keyvaninia, G. Roelkens, D. Van Thourhout, D. J. Thomson, F. Y. Gardes and G. T. Reed, "10 Gb/s Integrated Tunable Hybrid III-V/Si Laser and Silicon Mach-Zehnder Modulator,” ECOC 2012. 